\title{
Descriptive standards and collection management software for documentary heritage management: attitudes and experiences of information professionals
}

Collection management software

\author{
Chelsea Renshaw \\ Library, Walsh Memorial Library, Museum of Transport and Technology, \\ Auckland, New Zealand, and \\ Chern Li Liew \\ Victoria University of Wellington, School of Information Management, \\ New Zealand
}

\begin{abstract}
Purpose - This paper aims to examine the attitudes and experiences of information professionals with descriptive standards and collection management systems (CMSs) used for managing documentary heritage collections held by cultural heritage institutions in New Zealand (NZ). The aim is that such insights will inform decision-making around promoting documentary heritage collections discoverability and accessibility, in terms of advocating for appropriate system requirements when procuring or updating CMSs, and application of descriptive standards.

Design/methodology/approach - A qualitative design was applied to investigate the attitudes and experiences of information professionals working in libraries, archives and records management institutions, museums and public galleries. Data was collected through semi-structured interviews with thirteen participants who worked across ten different cultural heritage institutions.

Findings - The findings reveal that variances among metadata in libraries, museums, public galleries, archives and records management institutions continue to lead to challenges around discovery and access of documentary heritage. If opportunities for connecting documentary heritage collections in the age of linked data are to be realized, the sector needs to work collectively to address these variances along with consideration of the CMSs used. The study findings highlight issues currently affecting the NZ cultural heritage sector goal to make collections discoverable and more widely accessible.

Originality/value - The findings highlight a need for deeper research into CMSs used by the cultural heritage sector as these systems have an impact on metadata management including constraining the application of appropriate descriptive standards for documentary heritage collections.
\end{abstract}

Keywords Metadata, Collection management software, Cultural heritage institutions,

Descriptive standards, Documentary heritage, Libraries, Archives, Museums, Records management, Public galleries

Paper type Research paper

\section{Introduction}

Galleries, libraries, archives, museums and records management institutions (GLAMRs) share a common responsibility of curating and providing access to documentary heritage

Global Knowledge, Memory and Communication c) Emerald Publishing Limited 2514-9342
Received 26 August 2020 Revised 30 October 2020 Accepted 4 November 2020 
collections along with their metadata. Changing information needs brought about by the Web environment have led to a push for more shareable metadata (Mitchell, 2013; Riley and Shepherd, 2009; Shreeves et al., 2006). The choice and use of collection management systems (CMSs) by GLAMRs have a significant impact on the management of documentary heritage collections as they are increasingly being used to enable the creation and the hosting of metadata. Likewise, descriptive standards support interoperability and discoverability of collections and items by providing a structure for how metadata are created and managed.

\section{Motivation for study}

Shareable metadata is commonly cited as an enabler for the wide sharing of documentary heritage collections. However, as pointed out by Han et al. (2009), research addressing how curators adapt or customise metadata standards for a local setting or the degree to which this impedes interoperability remain scant. A decade later, this remains the case. Studies have also suggested that CMSs have a role to play in improving interoperability and discoverability of collections and items (Jehly, 2013; Lim and Liew, 2011; Shreeves et al., 2006). Katre (2011) suggests a need to be more critical of the systems that are marketed as all-inclusive or claiming to be broadly standards-compliant across the sector. Shreeves et al. (2006) suggest that CMSs need to support a modular and increasingly multi-standards based approach. A multistandards environment is integral for the management of documentary heritage collections that are held by a wide range of GLAMR institutions that adopt different CMSs.

There is however, no scholarly research that has focused on examining the impact CMSs have on cultural heritage institutions' selection and use of descriptive standards. Descriptive standards and CMSs have not been investigated concurrently, despite being positioned as significant in the digital preservation triad (Corrado and Moulaison Sandy, 2017). The objective of this research is to address this gap and to provide insights into issues that have an impact on the creation and management of descriptive metadata for documentary heritage collections by also bringing CMSs into the discourse. This study investigates the experience and attitude of staff working in New Zealand (NZ) GLAMR institutions regarding:

- the selection and application of descriptive standards for documentary heritage collections; and

- CMSs used for managing documentary heritage materials in their institutions

The aim is that such insights will inform decision-making around promoting documentary heritage collections discoverability and accessibility, in terms of advocating for appropriate system requirements when procuring or updating CMSs, and application of descriptive standards. A collective understanding promotes new ways for the cultural heritage sector to think about potential solutions given that the institutions in this sector share the common goals of metadata interoperability and collection discovery) even if they function in different settings (e.g. a lending library, a reference archive, a museum or gallery space).

\section{Literature review}

This literature review brings together related works that address the common goals of collection discoverability and accessibility shared by GLAMRs and their approaches to metadata.

\subsection{Documentary heritage}

Documentary heritage is defined by the United Nations Educational, Scientific and Cultural Organization's (2017) "Memory of the World" project as having the following 
characteristics: mobile; consisting of signs/codes, sounds and/or images; can be conserved (the supports are inert elements); can be reproduced and transported; and is the result of a deliberate documentation process. According to Prodan (2013), its significance is twofold. Firstly, the information value of documentary heritage can be instrumental in shaping knowledge societies by offering context to the past. Of equal importance is the information carrier that can inform an understanding of how societies frame and disseminate knowledge in relation to the distribution of power.

Material appraised according to these two measures of significance distinguishes it from other resources because the objective is often perpetual preservation (of object and context) at the point of entry into the institution. Pitti (1999) calls this difference in the nature of what is remembered, as well as who it is on behalf of. This necessitates the need for more detailed metadata and catalogue records that include information about access conditions, provenance, as well as biographical and scope and contents listings (Chang, 2000). Given the carrier is also of equal importance, (such as object-biography metadata) are added to this list, especially when dealing with material such as prints or drawings. Documentary heritage collections thus contend with interdisciplinary management through being located at the intersection of library and archival science, museology, and use as aesthetic/art objects.

\subsection{Managing documentary heritage collections - challenges with convergence}

Several studies have focused on the problematic effect of the convergence of GLAMR institutions on the management of collections, highlighting the post-convergence difficulties of representing an archival context. Robinson's (2012) comparison of the different epistemological frameworks of libraries, archives and museums evidence the different ways historical knowledge is produced by each of these domains. It is argued that comparative delineation of the way collection information is packaged by each institution type is necessary to assess the long-term implications of convergence on managing culturally significant materials.

A study by Wellington (2013) found that archival collections faced dismemberment by curators seeking to manage objects according to museum frameworks. This was linked to a lack of understanding of the archival domain at management level, with the organisational structure misaligning recordkeeping positions by separating archivist and record manager. Similar findings by Jehly (2013) evidenced frustrations with inappropriate systems for the collection management of archives in a museum setting. Further consequences of this are reported in a study examining how systems and practice aid the emergence of evidence. Sanderson (2017) demonstrated that the positioning of photographs as aesthetic objects divorced them from their provenance and resulted in finding aids that highlighted them as orphans in a collection, further divorcing them from other collections by the same photographer held by another institution.

\subsection{GLAMR metadata - diverging standards cultures}

The above cases of "convergence" hint at the impact on documentary heritage collections when not managed according to a framework that best suits the context of the object. Partly driven by the diverging requirements for physical collection management, different GLAMR metadata cultures have concurrently developed. Roel (2005) argues that the different metadata cultures among libraries, archives and museums have emerged from differences between documentation and organisation of objects, and have presented challenges for representing different types of material across the sector. Waibel and Erway (2009) view this fragmentation in the physical world necessary for adequate care of objects, 
but argue it is at odds with user expectations for discovery in a single search environment online.

Roel(2005) also addresses the harvesting project Open Archives Initiative Protocol for Metadata Harvesting (OAI-PMH) for bridging metadata differences between institution types. However, Riley and Shepherd (2009) argue that OAI-PMH alone is not sufficient for wider, open sharing of archival metadata because of the limited fields in the Dublin Core standard, which can lead to overstuffing of metadata records or preclude the inclusion of fields holding contextual metadata. For Corrado and Moulaison Sandy (2017), this provenance information provided by contextual metadata is essential for researchers to access and to have details about the context and authenticity of collections.

\subsection{Shaping a new approach to the application of standards and education}

With diverging metadata cultures, there is a common theme in the professional discourse and literature with regard to the need for the sector to shape an approach to descriptive practice that streamlines creation through improved use and awareness of the various descriptive standards. Given and McTavish (2010) suggest that a reworking of education may serve to bridge diverging skillsets between information professionals and engender better understanding for each domain's epistemological framework.

Duff et al. (2013) cite a lack of broad knowledge across standards or the skillset to converge varying metadata across access systems. Elings and Waibel (2007) suggest a realignment of existing standards to meet object-based needs rather the current practice of developing according to domain-based differences in the approach to description. Waibel and Erway (2009) recommend cross-pollination of existing standards, using authority controlled terminologies to point to a single authority record, irrespective of the domain of information science from which the description emerges. Banush (2010) argues that future skills for cataloguers will include broader knowledge of an array of metadata standards and schemas.

\subsection{Standards - universalism or marginalisation}

A standardised approach to describing collections have been critiqued for marginalising indigenous knowledge, as well as queer identities by reinforcing binary gender classifications (Billey et al., 2014; Montenegro, 2019). Montenegro (2019) sees standardised metadata creation as is inherently problematic for reinforcing Western paradigm of knowledge creation. Standardisation is argued to be a form of universalism not suited to upholding indigenous data sovereignty. Montenegro addresses this in relation to the creator and rights management fields in content standards, and highlights the privileging of Western concepts of intellectual property ownership.

Another critique by Billey et al. (2014) considers queer theory in context with the RDA directive to record gender. It argues that a fixed understanding of gender is privileged by only offering choices "male", "female" or "not known." The rule has since been lobbied and changes made by the RDA steering committee. Another issue resulting from current approaches to description has seen end-users isolated from archival materials. Trace and Dillon (2012) argue that the genre of finding aid is a communication tool that privileges use by archivists and researchers already familiar with the genre.

\subsection{Approaches to creating metadata}

The share-ability and versatility of metadata for documentary heritage collections is also influenced by diverging approaches to describing archival materials within the domain of information sciences. Wells (2012) discusses the ways differing interpretations of archival 
theory can result in metadata variances. One suggestion is the need for institutions to work more collaboratively in the creation and use of authority-controlled terminologies. This is at odds with the traditionally unstructured approach to describing archival materials.

Stead and Liew (2020) addressed the description of editorial cartoons and highlighted a common debate in the literature between the relative values associated with the use of controlled terminology versus free text fields. In a similar tenet, Parilla et al. (2017) examined the description of science notebooks and highlighted researcher expectations for access, which called for highly detailed item-level record description, did not fit well with existing standards for archival description of field note collections.

Existing metadata has also been evaluated to explore the possibilities of interoperability in the networked age. Gracy's (2015) research examined interoperability by exploring existing archival metadata elements and mapped components from EAD and MARC records to existing linked data vocabularies. This study counted the rates of success, highlighting EAD as the more fruitful standard for exploiting linked data opportunities. Gracy argued that continued practice of including rich descriptions in free-text fields that lack semantic definition would perpetuate the isolation of archival metadata. Rolan (2015) meanwhile, argued for interoperability to be designed into systems to facilitate appropriate data entry at the point of capture.

The pressure of "more product, less process" also has an impact on the creation of descriptive metadata (Riley and Shepherd, 2009). Riley and Shepherd explained the approach involves working to a level of description that matches the resourcing assigned to a particular collection, significance-dependent, rather than a monolithic descriptive standard to which all collections must comply with. Ascher and Ferris (2012) highlighted the process of developing collection surveys for the Association of Research Libraries to expose hidden special collections and produce more product. A set of descriptive elements with minimalmaximal metadata requirements was proposed.

\subsection{Collection management system research}

Evans (2014) argues that software are important to the descriptive frameworks utilised because it is their structure and functionality that have an impact on what and how metadata can be captured. Lim and Liew (2011) find that proprietary software have an impact on metadata creation. Their findings reveal that the use of proprietary systems, which come packaged with pre-existing metadata fields, shaped a systems-based rather than a compliant, standards-based approach to metadata management. Shreeves et al. (2006) highlight a need for support for CMS users to drive development and support of more standards, regardless of whether proprietary or open source software is being employed.

McKemmish et al. (2020) see an opportunity for co-designing digital technologies with users, especially those disadvantaged through maintaining practices that perpetuate a form of colonial power dynamics. This idea of co-designing systems is in line with the Digital Curation Centre's concept of a digital repository, seen as being equally about the people and processes as it is about software or hardware.

\section{Research design}

A qualitative methodology was applied and semi-structured interviews were conducted to investigate the attitudes and experiences of information professionals working in libraries, archives and records management institutions, museums and public galleries. Qualitative research places emphasis on the individuals and addresses the role that context and relationships have on forming thoughts and behaviours (Roller and Lavrakas, 2015). This approach was chosen as this research placed professional practice in the context of 
information professionals' unique perspectives. The aim was to collect data that would contribute to a rich understanding around the use of descriptive standards and CMSs for documentary heritage management.

\subsection{Assumptions and limitations}

Corrado and Moulaison Sandy (2017) identify four types of metadata - administrative, technical, descriptive and structural. This research focuses on descriptive metadata as they are the foundation that enables users to find, identify and access digital objects within a system and across different platforms (Corrado and Moulaison Sandy, 2017). This research also acknowledges that no single descriptive metadata standard can work for all digital collections (Han et al., 2009). We also acknowledge that standardisation by nature is linked to a concept of universal representation, a Western framework for managing collections. This has considerable limitations on NZ GLAMRs operating in a bicultural context, as these institutions have an obligation to respect Te Tiriti O Waitangi (www.treaty2u.govt.nz/thetreaty-up-close/treaty-of-waitangi/), with an acknowledgement that universality may not be an appropriate framework for the management of indigenous cultural heritage resources.

\subsection{Sampling}

The study participants were recruited through snowball sampling (Handcock and Gile, 2011). Thirteen participants who worked across ten different GLAMR institutions in NZ were interviewed. For confidentiality purposes, all data was de-identified, including the names of the CMSs concerned due to the relative small study sample, to avoid the possibility to identify the institutions. Table 1 outlines the interviewees' profiles.

Given the small sample, it is not our aim to claim generalisability of our findings. We also acknowledge that institutions of different sizes, with levels of resourcing would have unique challenges when it comes to metadata standardisation and CMS usage. Nevertheless, this research offers a starting point to examine metadata standardisation and CMS application as an integrated approach to promote collection discoverability and accessibility, offering insights that could inform how the sector as a whole or institutions advocate for CMS system requirements and appropriate descriptive standards.

\subsection{Ethical considerations}

Roller and Lavrakas' (2015) guidance on positive engagement was considered in the semistructured interviews. Recruitment of participants were undertaken with care to avoid coercion and participants were warned of the risk of potential identification due to the scope and specific context of the research. Each participant was offered a copy of the transcript of their interview, a summary of the findings and the full report.

Approval to conduct the study was granted by the Victoria University of Wellington Human Ethics Committee. A copy of the human ethics application (Application ID: 27988) can be obtained from the corresponding author.

\subsection{Data collection and data analysis}

The semi-structured interview method was chosen as it enabled the elicitation of rich data to understand individual's experiences and perspectives (Hammersley and Campbell, 2012) and provided interviewees room to reflect on their individual experiences (Brinkmann, 2013). These were important facets of this research. The semi-structured interview guide is presented in the Appendix. 


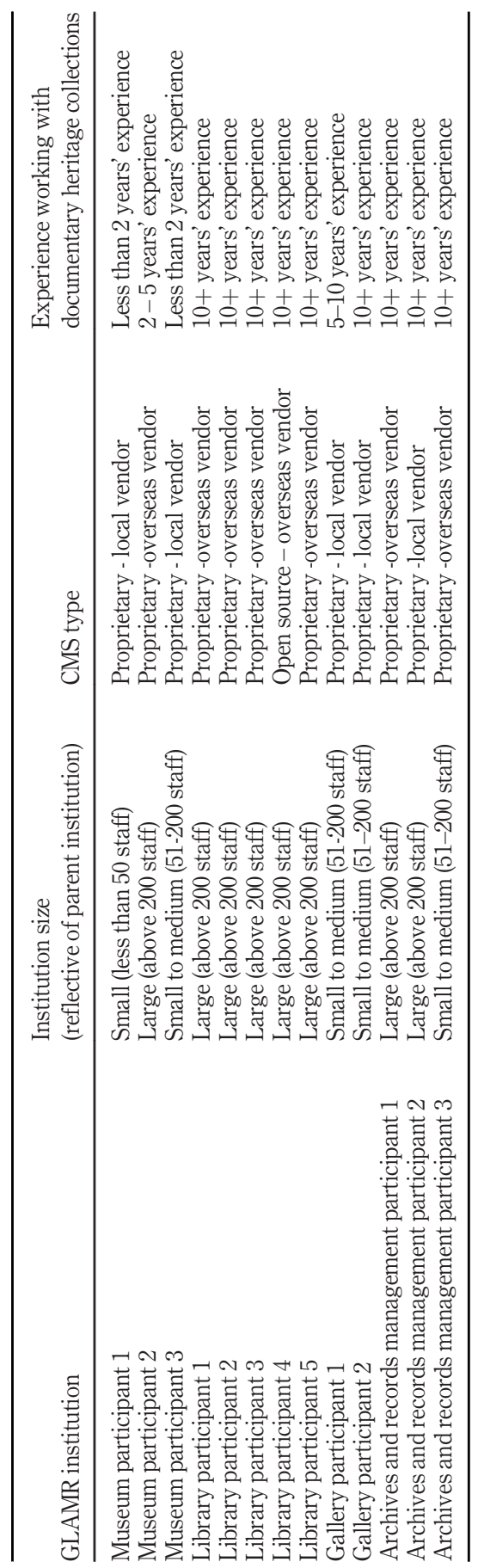

Collection management software

Table 1. Participants' profiles 
The interview transcripts were coded according to whether it was an attitude or experience relating to either descriptive standards or CMSs. These were further organised under the sub-codes suggested by Leedy and Ormrod (2016): process, action, emotion, evaluation, belief, value. This helped in the analysis to identify commonalities and comparisons across the participants' experiences and attitudes.

\section{Findings}

\subsection{Descriptive standards}

5.1.1 Perceived value of descriptive standards. When questioned about the importance of descriptive standards, interviewees reported that they were critical in aiding discoverability and maintaining data integrity, particularly across collections of documentary heritage. Museum participant 3, though agreed on the importance of standards, questioned the way standards were defined however, and viewed them more as guidelines citing that standards require a lot of time and resource.

Participants also expressed concerns about the neutrality of descriptive standards. Six participants voiced concerns ranging from who decided on standards and for whom, and how they were alienating users by being too laden with jargon or too niche. For Library Participant 4, who worked in an archival unit within a library, user expectations for access by researchers and students were observed:

The other thing I might say here is, as archivists, we spend a lot of time worrying about the hierarchical arrangement, which works, but what a lot of researchers just want is the item they can get and look at.

On access, Library Participant 2 noted a tension between being committed to creating linked open data for collections and being a custodian of collections with cultural significance for Māori (NZ indigenous people):

Who decides what the controlled authorities are going to be, who is in control? Is it the source community or is it an isolated group of white, middle class librarians?

These views emerged from experience on desk shifts, and by reflection on the intimidation felt by iwi (tribes) or whānau (extended families) when it came to navigating systems and wading through data to access records. Similar views were voiced by Archives and Records Management Participant 3:

Making sure [records] are created appropriately with all of the impacted people having a right to influence how they're created, managed and used in the future. [Reflecting on priorities for managing documentary heritage collections].

5.1.2 Experience with implementation and application of descriptive standards. Eleven interviewees identified standards that informed their descriptive practice. These fell into the categories of content, value or structure standards. Eleven participants could also identify either policy or formal/informal process documentation they could consult or were currently involved in developing. However, the collective experience of the interviewees in our study highlight a lack of consistency and conformance across the sector. Different institutional contexts have an impact on the ability to standardise.

Library Participants 3 and 5 both pointed to the merger environment in which they were working as a significant challenge to the implementation of standards. Library Participant 3 pointed out the challenge in the consistent application of standards was largely due to the variance in descriptive practices in a merged environment, including in local adaptions, as 
well as misuse of standards. There was also the feeling by this participant that getting buyin from other staff members was a challenge:

Certainly in this building people have been here a long time and, you know, I'm not an archivist, I'm not a photograph specialist, so should I be telling people what they should be doing when that is their expertise?

Museum Participant 1 discussed a lack of staff when trying to fit standards in around business as usual. Museum Participant 3 expressed similar views, citing that many institutions did not have the time or resources to manage the ongoing requirements and maintenance of standards. Museum Participant 2 described the struggle for the institution's top-level management to see the value of standards with limited resources, suggesting competition with flagstaff projects.

There was also the feeling that certain descriptive standards did not meet the representational needs of the material in their custody. This was raised by five interviewees. Archives and Records Management Participant 1 felt that Dublin Core always need to be extrapolated for local settings that relates back to limitations reported by Lim and Liew (2011) and Riley and Shepherd (2009). Gallery Participant 1 and Museum Participant 2 gave examples of challenges representing the archival context of material using RDA in a MARC structured databases, leading to a potential overstuffing of notes fields such as the 500 field. For Museum Participant 2, there were particular concerns around their obligations to privacy and wanting to comply with the NZ Public Records Act as best practice. Library Participants 1 and 2 on the other hand, observed that an EAD-structured CMS is primarily designed for the description of finding aids, not item-level object description.

Two participants raised how information professionals have different disposition even within an institution. Library Participant 1 noticed this when using the catalogue as a searching tool pointing out that, although there are standards and rigorous rules, human differences still emerge. Archives and Records Management Participant 3 gave the example of different professionals either clumping or granularly splitting groups of records at the point of description:

An issue can arise that makes you think in a particular way and you might change the standard to make it work. That can influence how you do something in the office from then on and some things can be not really noticeable on a national level, so, sure you've got people checking at a national level but they're checking for accuracy, not really tendencies.

Another challenge to implementation and application that emerged from the data was the notion of process versus product when it comes to managing incoming collections and existing backlogs. Library Participant 3 voiced the challenges faced in terms of having to reach a balance between creating comprehensive metadata records versus catching up with backlog:

I find that balance between yes, we want to have some kind of standard, but in reality, if we can't keep up, how much do we put up for people to see. I don't know!

On products and processes, this participant also questioned the effectiveness of skeletal (minimal) description when it came to supporting user discovery. Concerns with backlogs were shared by six other participants. Four of them mentioned the importance of collection development for managing incoming collections to curtail further backlogging.

Wider sector leadership and collaborative work practices were also common mention in the interviews. Gallery Participant 1 commented on feeling very supported by the Art Libraries Society when it came to cataloguing documentary heritage, while Archives and Records Management Participant 1 said the Association of Local Government Information 
Managers were working towards a standardised set of metadata for members. Museum Participant 2, on the other hand, felt conflicted:

In terms of how cataloguing standards are working for institutions, I don't think there has been a lot of leadership around rare books and what to do [... . There is no consensus in the field.

\subsection{Collection management systems (CMSs)}

5.2.1 Attitudes towards collection management systems. Attitudes towards CMSs were most evident when it came to participants discussing the type of support they received or the lack of support, as well as development for the systems used, both from external vendors and within the institution. Experiences were dependent on the organisational structure and processes for the management of information technology employed by the institution.

Seven participants discussed direct experiences with software vendors, highlighting a mixture of attitudes. One participant in an archives and records management environment expressed concerns that vendor support has generally not been user-driven, but typically consisted of patches or version upgrades. Three other participants who worked across library, museum, and archives and records management contexts talked about the opportunity to submit development suggestions through vendor networking days or online forums. However, one participant had doubts in terms of how successful developments were:

As I say, it's very static. Once it's built, it's built. I'd like to see them offering new apps and things. - Archives and Records Management Participant 2

This participant expressed uncertainty on whether the lack of development was because they managed a relatively small amount of content made available to the public (fewer than 1000 records). This participant recalled two occasions of development requests that were not followed-up on by the vendor.

On the other hand, the participant working in an open-source CMS described support and development in very positive terms, calling it a community that is completely user driven. On one occasion, this library was able to piggyback off developments by another institution with more resource capabilities and enhanced the way collections were able to be shared with the public.

Five participants discussed how their CMS were supported in-house. Gallery Participant 2 reflected on how this had developed organically because of two staff member's expertise in the CMS and their helpfulness and proactive-ness when supporting other team members. For another participant, where the software support was managed completely in-house, the experience was undesirable after losing dedicated CMS specialists:

It's a matter of having IT people in the organisation who are able to respond quickly and to understand the system and what's for. -Archives and Records Management Participant 3

Similarly, Archives and Records Management Participant 1 felt privileged to have in-house support but also discussed challenges when communicating their needs to the IT unit. Interestingly, there was division in attitudes when it came to the benefits of having a local software provider, particularly in terms of the development and support of CMSs. Two participants felt that the locality of vendors enhanced the support you received:

Probably, I think, the fact that they're a local company is good for us as well. Just my experience with overseas vendors has been that, firstly, they're often creating product for a very large market of which you're a tiny, tiny part with maybe slightly different practices from the bulk of users. -Museum Participant 1 
This contrasted with the experiences of two other participants who felt that support from their local vendors was inhibited by scale and proximity of the working relationship:

I think the relationship is strong between them and our institution, it's company to company sort of thing. The scale thing, though, you could argue inhibits your ability to change the product and make the changes being requested. -Museum Participant 3

The variations across the data suggest that support and development could be an area that would benefit from further research, in part due to interviewee evidence showing their relationship to creating and managing metadata.

\subsection{Experiences with collection management systems}

The infrastructure of CMSs can be crucial in determining what descriptive standards are adopted by institutions. For Library Participant 5, standards were an important factor when procuring a new CMS:

Totally, you can import from MARC and it's based on DC. That was one of the selling points.

The CMS adopted by Library Participant 4 not only supported the DACS standard, but also included a rollover feature with pop-up boxes to guide users on how each field aligned with the standard. There was also evidence validating the need for further research into whether CMSs support a multi-standards operating environment. Seven participants in the study sample reported working with a CMS that had been structured to primarily manage material in a way that did not always suit the collections in their care.

Content can actually have multiple reasons for existing, but being able to clearly identify that is actually often restricted by the software that you're using. -Museum Participant 3

Among the interview data, restrictions emerged around the type of descriptive metadata that could be created within CMSs for managing documentary heritage. Two participants described the difficulties of capturing the archival context of material in notes fields using Library Management Systems as a CMS for documentary heritage. Museum Participant 2 highlighted the range of metadata that was felt to be compromised by the system's functionality:

With item level records, item level details like signatures and marks, condition, risk management, valuation, provenance, attaching the right paperwork to the records, having the right privacy settings for different things, image metadata like making sure that comes across clearly, data security, the things you'd associate more with a heavier archival system.

Challenges representing paper-based objects in an archival context were also noted. One participant highlighted how archival descriptive practice based around hierarchical arrangement presented challenges when describing singular objects:

For a sketchbook there will be a parent record that is the book and child records for each page in the book. There might even be grandchild records if there are two things stuck into one page of a book. You might have a record for each of those items that are stuck in. They would be under the child record for the page itself. It's impossible to avoid that, but if you can avoid it, you should because what we find with $\mathrm{EAD}$ is that it's difficult for users to navigate. -Library Participant 2

Another participant who worked at the same institution noted that working in a single system with colleagues from different backgrounds in education and sector experience presents challenges when it came to advocating for a more object-based approach to arrangement and description. An in-house working group for the CMS aided communication between colleagues. The interviewee concerned found this to be a valuable space for 
contribution and negotiation of such challenges. The same sentiment was voiced by Museum Participant 1 who thought that being open to each other's ways of working was a worthy payoff to have one CMS.

Archives and Records Management Participant 2 viewed large, proprietary CMSs as complicated, costly and beyond the requirements of most smaller institutions. Experience using a large proprietary system for metadata creation was also expressed by another participant as problematic:

I think one of the strengths of the software is that it doesn't tell you how you have to handle it, but one of the weaknesses of it in a small institution, with no standards, you've got ultimate freedom to do things different ways each time if you want and we have. -Museum Participant 1

Conversely, Library Participant 1, who worked in a much larger institution viewed the breadth of functionality in positive terms because of the ability to create richer records, which was seen to suit item level cataloguing for the material in this department's custody. Three other participants in large institutions also mentioned struggles with legacy metadata.

Two participants mentioned that the ability to make updates to legacy data was hindered as the CMSs they used meant it was difficult to make bulk changes to metadata. Library Participant 3 felt that controls over bulk edits were not robust enough to confidently make changes en masse. Museum Participant 2 mentioned a lack of support for editing legacy data and described feeling more pressure to do what was described as physical, visible work. It was felt that the cleaning up of the metadata of 500 records did not get the same response. Although only raised by two participants, the significance of managing legacy data was tied into future preparedness of metadata.

Three interviewees also raised use of te reo Māori in software highlighting challenges and workarounds. A common issue expressed was the display of macrons:

We've had a fix that has worked in some aspects and not in others. It can handle macrons to a degree, but it's a workaround. It wasn't built in from the start, so it doesn't work brilliantly. Archives and Records Management Participant 3

\section{Discussion}

\subsection{Challenges to metadata standardization}

Findings from this study are consistent with many of the challenges discussed in the literature. Lim and Liew (2011) have highlighted the limitations of Dublin Core, the lack of resourcing in smaller institutions as a significant issue for creating interoperable metadata for digital surrogates, as well suggesting challenges with proprietary CMSs for creating appropriate metadata. Experiences from interviews conducted in this study add to Lim and Liew's (2011) findings by showing that standards implementation can become an issue, irrespective of institution size, when their value is not translated because of different institutional contexts at play that are also impacted by CMSs. This also reflects the point made by Corrado and Moulaison's (2017) that technology enables and helps the creation of descriptive metadata, but it is ultimately a management decision as to the level or amount that is deemed necessary. Interviewees provided examples they had encountered that challenged the implementation, maintenance and selection of appropriate descriptive standards. They believed these affect the quality of metadata meant to support discovery and access to documentary heritage collections.

The quality of metadata to support user discovery and access is only going to become more salient in NZ GLAMRs following the Ministry of Business, Innovation and 
Employment's (MBIE) review of the NZ Copyright Act 1994.In their submissions to the Issues Paper by MBIE supporting associations representing the sector, including the Library and Information Association of New Zealand Aotearoa (2019) and Museums Aotearoa (2019), rallied for a fair use framework and further exceptions for reuse of collections respectively. With the upcoming possibility to increasingly share collections in an online environment, it will become more important to create descriptive metadata that is both consistent and conforms to standards, as well as the need to have an informed understanding of how users, including aggregators, can work with metadata.

In the examples discussed by the interviewees in our study sample, challenges to standardization emerged from different approaches or interpretations of descriptive standards. These were linked to practices applied across sites, despite professional's creating metadata the collections online platforms hosted by their respective institutions. Wells (2012) acknowledges the validity of information professionals' differing interpretations of collections that may result in differences between a set of records' descriptions. However, there is the question of how these differences may affect user understanding of collections, along with how local adaptions could be impeding interoperability. This becomes an issue for metadata aggregators, where the most important factor is being able to understand the descriptive approach that has been applied, as well as the metadata standard chosen to express that approach (Riley and Shepherd, 2009). It would be valuable to examine the ways in which institutions are capturing and documenting their workflows, and how such documentation informs and affect decisions.

There are also questions around the document-centric approach to describing collections in an archival context. The traditional hierarchical approach was questioned by three participants in terms of the extent to which it met user expectations to discover and access collection items across different platforms. This hierarchical approach is currently supported by descriptive standards including DACs and $\operatorname{ISAD}(\mathrm{G})$ and EAD. It is applied to capture the context of records creation and use. Riley and Shepherd (2009) view this type of finding aid as a rich archival narrative but argue the hierarchical approach is at odds with most aggregators' record-centric approach to harvesting metadata, making archival metadata harder to share. Daniels and Yakel (2010) suggest developing heuristics for novice engagement with finding aids but perhaps user expectations associated with the discovery of documentary heritage collections that have heavier archival context may be further explored. A cross-pollinated standards environment could be supported by innovative metadata visualization that take descriptive standards as a starting point to support multiple user understandings of collections.

\subsection{Impact of collection management systems}

This study provides further insight into information professionals' attitudes and experiences with CMSs. The most significant is the impact of CMSs on metadata management, particularly in terms of potentially constraining their selection and application of appropriate descriptive standards for their collections. Interviewees suggest that descriptive metadata management is not a static activity but an aspect that should evolve with changing user expectations for discovery and access.

Another significant finding that merits further examination concerns how CMS structure could support a range of descriptive standards, which could be addressed concurrently with an evaluation of system requirements already communicated to CMS vendors. Awareness of how CMSs support affect or restrain the application of descriptive standards is relatively low among the interviewees. This shows the continued relevance of Lim and Liew's (2011) findings that information professionals often base their metadata creation according to 
proprietary systems that have pre-defined metadata fields rather than doing so with interoperability, discoverability and long-term preservation at the fore of their decisions.

\subsection{Advocacy}

A few interviewees also raised the importance of indigenous self-determination with regard to how documentary heritage is described, organised and preserved. McKemmish et al. (2020) highlight the need for transformation to incorporate indigenous worldviews in archiving and recordkeeping. For Ballantyne (2019), the transient nature of collections can make them a "springboard for new processes of collection-building and story-telling [...] to achieve a greater degree of intellectual and cultural autonomy" (p. 5). To achieve greater degrees of intellectual and cultural autonomy, evolving and inclusive description must include significant participation from the groups the records concern.

Evidence from this research suggest that the infrastructure of certain CMSs can be a barrier to an institution's effort to decolonize their item description. Ballantyne (2019) highlights power in the role of arranging archives. Given that collections are non-static sites, further research may focus the ways in which CMSs have impact on the description of indigenous knowledge collections and appropriate improvements. McKemmish et al. (2020) see opportunity in co-designing technological infrastructure as a way forward to end the perpetuation of visiting trauma upon powerless participants in records.

\section{Conclusion and area for further research}

With the proliferation of digital technologies, GLAMRs are facing increased pressure to converge or reconcile their different descriptive metadata. This is largely driven by user expectations for discoverability of collections and items across platforms and systems. This study has evidenced the perpetuation of issues surrounding metadata creation and management in the cultural heritage sector. Variances between metadata continue to lead to challenges around discovery and access. If opportunities for connecting documentary heritage collections in the age of linked data are to be realized, GLAMRs need to work collectively to address how the different institutions can work to consistent standards level of description while enabling flexibility within specific contexts when necessary, with appropriate support from the CMSs they employ.

One area for further research is to examine the challenges raised by several interviewees in this study with regard to examining the education pathways across the sector, with regard to descriptive frameworks and practices. It can also be argued that if conformance to standards is essential for creating shareable metadata, this must be balanced with effective approaches to manage the scale of collections according to a reasonably resourced framework for care. The tension between products and processes has been highlighted by the interviewees. Understanding the scale and extent of backlogged documentary heritage collections may be a departure point from this research to provide tangible evidence behind the need to secure improved resourcing.

Another research avenue emerging from this study is the need for further insights into the impact descriptions and CMSs have on the discoverability and access to minority cultures and indigenous heritage collections. It would be timely to address how minority communities might be marginalized by limitations in current descriptions that perpetuate a form of colonialism and how this can be addressed through decision-making with regard to institutional descriptive approaches and practices, and how the design of CMSs could be an important aspect of the resolution. 


\section{References}

Collection

Ascher, J.P. and Ferris, A.M. (2012), "Collection-level surveys for special collections: coalescing descriptors across standards”, The Journal of Academic Librarianship, Vol. 38 No. 1, pp. 33-41, doi: 10.1016/j.acalib.2011.11.006.

Ballantyne, T. (2019), "From colonial collection to tribal knowledge base: Herries beattie, ngāi tahu whānui and the many lives of an archive", Journal of Colonialism and Colonial History, Vol. 20 No. 2.

Banush, D. (2010), "Cooperative cataloging at the intersection of tradition and transformation: Possible futures for the program for cooperative cataloging", Cataloging and Classification Quarterly, Vol. 48 No. 2-3, pp. 247-257.

Billey, A., Drabinski, E. and Roberto, K.R. (2014), "What's gender got to do with it? A critique of RDA 9.7”, Cataloging and Classification Quarterly, Vol. 52 No. 4, pp. 412-421.

Brinkmann, S. (2013), Qualitative Interviewing, Oxford University Press, New York, NY, doi: 10.1093/ acprof:osob1/9780199861392.001.0001.

Corrado, E.M. and Moulaison Sandy, H. (2017), "Digital preservation for libraries", Archives, and Museums, Rowman and Littlefield Publishers.

Duff, W.M., Carter, J., Cherry, J.M., MacNeil, H. and Howarth, L.C. (2013), "From coexistence to convergence: studying partnerships and collaboration among libraries, archives and museums", Information Research: An International Electronic Journal, Vol. 18 No. 3.

Elings, M. and Waibel, G. (2007), "Metadata for all: descriptive standards and metadata sharing across libraries, archives and museums", First Monday, Vol. 12 No. 3.

Evans, J. (2014), "Designing dynamic descriptive frameworks", Archives and Manuscripts, Vol. 42 No. 1, pp. 5-18.

Given, L. and McTavish, L. (2010), "What's old is new again: the reconvergence of libraries, archives, and Museums in the digital age", The Library Quarterly, Vol. 80 No. 1, pp. 7-32.

Gracy, K.F. (2015), "Archival description and linked data: a preliminary study of opportunities and implementation challenges", Archival Science, Vol. 15 No. 3, pp. $239-294$.

Hammersley, M. and Campbell, J.L. (2012), What is Qualitative Research?, Bloomsbury Publishing, Huntingdon.

Han, M.-J., Cho, C., Cole, T.W. and Jackson, A.S. (2009), "Metadata for special collections in CONTENTdm: how to improve interoperability of unique fields through OAI-PMH", Journal of Library Metadata, Vol. 9 Nos 3/4, pp. 213-238.

Handcock, M.S. and Gile, K.J. (2011), "Comment: on the concept of snowball sampling”, Sociological Methodology, Vol. 41 No. 1, pp. 367-371.

Jehly, C. (2013), Mixed Material: Investigating the Management of Archives in New Zealand Museums, Master of Information Studies, Victoria University of Wellington.

Katre, D. (2011), "Digital preservation: converging and diverging factors of libraries, archives and museums - an Indian perspective", IFLA Journal, Vol. 37 No. 3, pp. 195-203, doi: 10.1177/0340035211418728.

Leedy, P.D. and Ormrod, J.E. (2016), Practical Research: planning and Design/Paul D. Leedy, Late of American University, Jeanne Ellis Ormrod, University of Northern CO (Emerita), 11 ed., Pearson, Boston.

Library and Information Association of New Zealand Aotearoa (2019), "Submission on consultation document: Implementation of the trans-pacific partnership intellectual property chapter", available at: www.mbie.govt.nz/dmsdocument/1911-lianza-intellectual-property-tpp-sub-pdf

Lim, S. and Liew, C.L. (2011), "Metadata quality and interoperability of GLAM digital images", Aslib Proceedings, Vol. 63 No. 5, pp. 484-498.

McKemmish, S., Bone, J., Evans, J., Golding, F., Lewis, A., Rolan, G. and Wilson, J. (2020), “Decolonising recordkeeping and archival praxis in childhood out-of-home care and indigenous archival collections", Archival Science, Vol. 20 No. 1, pp. 21-49. 
Mitchell, E. (2013), "Metadata developments in libraries and other cultural heritage institutions", Library Technology Reports, Vol. 49 No. 5, pp. 5-1012.

Montenegro, M. (2019), "Subverting the universality of metadata standards", Journal of Documentation, Vol. 75 No. 4, pp. 731-749.

Parilla, L.L., Morgan, R. and Fidler, C. (2017), "Excavating archival description: from collection to data level”, Digital Library Perspectives, Vol. 33 No. 3, pp. 195-202, doi: 10.1108/DLP-11-2016-0043.

Pitti, D. (1999), "Encoded archival description: an introduction and overview", New Review of Information Networking, Vol. 5 No. 1, pp. 61-69.

Prodan, A.C. (2013), "Documentary heritage, digital technologies and the dissemination of knowledge", " In Albert, M.-T., Bernecker, R. and Rudolff, B. (Eds), Understanding Heritage: Perspectives in Heritage Studies, Walter de Gruyter GmbH, Berlin-Boston, pp. 155-168.

Riley, J. and Shepherd, K. (2009), "A brave new world: archivists and shareable descriptive metadata", The American Archivist, Vol. 72 No. 1, pp. 91-112.

Robinson, H. (2012), "Remembering things differently: museums, libraries and archives as memory institutions and the implications for convergence", Museum Management and Curatorship, Vol. 27 No. 4, pp. 413-429.

Roel, E. (2005), "The MOSC project: Using the OAI-PMH to bridge metadata cultural differences across museums, archives, and libraries", Information Technology and Libraries, Vol. 24 No. 1, pp. 22-24.

Rolan, G. (2015), "Towards archive 2.0: issues in archival systems interoperability", Archives and Manuscripts, Vol. 43 No. 1, pp. $42-60$.

Roller, M.R. and Lavrakas, P.J. (2015), Applied Qualitative Research Design: A Total Quality Framework Approach, Guilford Publications, New York, NY.

Sanderson, K.M. (2017), "Digital materiality, heritage objects, the emergence of evidence, and the design of knowledge enabling systems", Doctoral Thesis, Victoria University of Wellington, available at: https://library.victoria.ac.nz

Shreeves, S.L., Riley, J. and Milewicz, L. (2006), "Moving towards shareable metadata”, First Monday, Vol. 11 No. 8,.

Stead, O. and Liew, C.L. (2020), "Editorial cartoon collections: a review of indexing challenges", Aslib Journal of Information Management, Vol. 72 No. 3,

Trace, C.B. and Dillon, A.J.A.S. (2012), "The evolution of the finding aid in the United States: from physical to digital document genre", Archival Science, Vol. 12 No. 4, pp. 501-519.

United Nations Educational, Scientific and Cultural Organization (2017), "What is documentary heritage?", available at: www.unesco.org/new/en/santiago/communication-information/memoryof-the-world-programme-preservation-of-documentary-heritage/what-is-documentary-heritage/

Waibel, G. and Erway, R. (2009), "Think globally, act locally: library, archive, and museum collaboration", Museum Management and Curatorship, Vol. 24 No. 4, pp. 323-335.

Wellington, S. (2013), "Building GLAMour: converging practice between gallery, library, archive and museum entities in New Zealand memory institutions", Doctoral Thesis, Victoria University of Wellington, available at: https://library.victoria.ac.nz

Wells, E. (2012), "Related material - the arrangement and description of family papers", Journal of the Society of Archivists, Vol. 33 No. 2, pp. 167-184. 
- What is your current role and how long have you been in it?

- Tell me a little bit about your background working in the galleries, libraries, archives, museums and records management (GLAMR) sector.

- How long have you been working specifically with documentary heritage collections? What types of collections do you have experience with?

- What do you see as the most important priorities for managing documentary heritage collections? Why do you think these are priorities?

- How familiar are you with GLAMR standards for managing documentary heritage collections? What is your experience applying these standards?

- How important do you think standards-based cataloguing is? How well do you think existing standards are working for describing documentary material?

- How is your institution looking to implement standards based cataloguing? What are some of the resource and time constraints you have faced which have precluded their application? (***Question posed to interviewee who worked in an institution that was at the beginning stage of developing processes for standardisation at the time of this research)

- What cataloguing standards has your institution implemented for describing documentary heritage collections? Can you give examples of your practice? Do you think it is a priority for your organisation?

- Can you outline any policies and processes your institution has put in place that support standards-based description of documentary heritage? For example, an in-house cataloguing manual referring to standards.

- Do you think the existing organisational policies and processes encourages standardsbased cataloguing in your institution? What about the CMS - Does this support standards-based cataloguing?

- Briefly describe the tasks you perform as part of your role with regard to the CMS.

- What are some of the challenges you have encountered when describing documentary heritage collections in CMSs? Can you talk through some examples?

- What do you think is working well with the current CMS you are using for managing documentary heritage collections? What is not working well?

- Can you describe your experience with the CMS vendor? For example, what support do they offer you as a client? What other services or support are desirable?

- What other CMSs have you had experience with? How do these compare?

- Is there anything else that you would like to comment on?

Corresponding author

Chern Li Liew can be contacted at: chernli.liew@vuw.ac.nz

For instructions on how to order reprints of this article, please visit our website:

www.emeraldgrouppublishing.com/licensing/reprints.htm

Or contact us for further details: permissions@emeraldinsight.com 